\title{
DNA aptamers in COVID-19 research
}

\author{
Sanya Jain ${ }^{1}$, Somesh Pratap Singh², Chaithra Mayya ${ }^{3}$, Sharmistha Majumdar ${ }^{3}$ \\ and Dhiraj Bhatia ${ }^{3, *}$ \\ ${ }^{1}$ Cognitive Science Discipline, ${ }^{2}$ Mechanical Engineering Discipline, \\ ${ }^{3}$ Biological Engineering Discipline, Indian Institute of Technology Gandhinagar, Palaj, Gandhinagar 382 355, India
}

\begin{abstract}
Novel coronavirus (SARS-CoV-2), a variant of the severe acute respiratory syndrome (SARS) family has claimed around 1 million lives and more than 33 million people worldwide have been infected. It has been declared a pandemic by the World Health Organization. COVID-19 is transmitted mainly through aerosol droplets from patients (both asymptomatic and symptomatic) to healthy people. Its high rate of transmission demands a quick and early diagnosis of patients followed by urgent quarantine of those affected. Since the SARS-CoV-2 virus is mutating, it is of utmost importance to develop a quick diagnosis against it. The current techniques use either PCR-based methods or antibody-based ELISA methods for diagnosis, which are both time-consuming and expensive. This is the biggest impediment in large-scale diagnosis of COVID-19. Multiple biosensors based on antibodies and aptamers have been reported and tested. Aptamers seem much more lucrative due to ease of synthesis, cost-effectiveness and extremely high degree of sensitivity in terms of detection, less immunogenicity and robustness to modifications. We present the history and characterization of aptamers, their selection strategies and applications to multiple viruses such as HIV, HCV and SARS-CoV. However, to date, no aptamers have been designed against any of the protein components or the genomic RNA of SARS-CoV-2. Based on the success of aptamers against many viruses, we argue for the future exploration of aptamers in the context of SARS-CoV-2 diagnostic testing.
\end{abstract}

Keywords: Biosensing, coat proteins, coronavirus, diagnostics, DNA aptamers, therapeutics.

THE outbreak of coronavirus (COVID-19) since December 2019 has plagued almost every part of the world. Approximately 33 million people have been infected with around 1 million deaths worldwide, and the cases are continuously increasing. In such outbreaks, quick and accurate diagnosis of affected cases, followed by quarantine of infected patients and medication-based treatments are the standard procedures adopted to prevent further spread.

The conventional methods used for diagnosis of a viral infection are: (i) isolation of virus and culture; (ii) detection

\footnotetext{
*For correspondence. (e-mail: dhiraj.bhatia@iitgn.ac.in)
}

of antibodies specific to the virus; (iii) detection of viral DNA or RNA by polymerase chain reaction (PCR) or RT (real time)-PCR respectively, using viral-specific primers $^{1,2}$. These methods, although used as standards, offer certain disadvantages which limit their usage for high-throughput screening. Virus separation and culture are laborious procedures to implement in routine clinical laboratories in many parts of the world ${ }^{2}$. Antibodies can be detected only after a period of 7-21 days post-infection, thus making this method inefficient for early diagnosis ${ }^{3,4}$. Further, cross-reaction of antibodies cannot be avoided due to the conserved sequences and structure similarities between different strains of viruses $^{5,6}$.

Detecting the nucleic acid of the virus by qRT-PCR is considered the gold-standard method so far $^{7}$. However, this method suffers in terms of the requirement of a high quantity of starting viral RNA or DNA to begin the PCR test. DNA-based oligonucleotides have served multiple purposes over time, ranging from applications in PCR and hybridization techniques to acting as drugs themselves. One of the most appealing and fascinating applications of DNA oligonucleotides is their ability to bind to specific target entities ${ }^{8,9}$. These sequences of DNA which can bind to different ligands with extremely high sensitivity are called aptamers (derived from the Greek word 'Aptus' meaning to bind) ${ }^{10}$.

This article focuses on multiple aspects of aptamers. First, we explain the basic concepts of aptamers and aptasensors, and compare them with protein-based antibodies. Next, we present a brief review of the selection strategies for aptamers. Further, we show how aptamers have been effective against a variety of viral and bacterial infections. Based on their diagnostic and therapeutic efficacy against the structural and accessory proteins of various viruses, we argue that aptamers must also be tested against the different proteins encoded by SARS-CoV-2.

\section{Aptamers}

Aptamers are single-stranded DNA or RNA nucleotide sequences that diagnose and inhibit the replication of a virus by intercalating with specific target molecules ${ }^{10}$. Naturally occurring nucleic acid sequences that can bind to target entities are mostly found in RNA. Naturally 
occurring DNA-based aptamers are rarely reported in the literature. However, DNA itself exhibits similar characteristics like RNA to recognize and bind to target entities. DNA offers multiple advantages over RNA in terms of its higher stability, cost-effective synthesis and minimum immunogenicity. Thus DNA is a good choice for developing aptamers for multiple applications ranging from tools for fundamental research to devices for biosensing and therapeutics ${ }^{11}$. The process of selection and identification of DNA or RNA-based aptamers from a large pool of single-stranded cDNA libraries is called systematic evolution of ligands by exponential enrichment $(\text { SELEX) })^{12}$.

Viruses like adenovirus and human immunodeficiency virus (HIV) encode a small structured RNA as their genetic material. This RNA is injected in host cells where it hijacks the host cell machinery for its own amplification and virion assembly. Aptamers offer a potential solution by mocking the viral RNA. The host proteins instead of binding to the actual viral RNA, bind to the decoy RNA. HIV has an RNA sequence called the TAR or trans-activation response. TAR RNA binds to the proteins Tat and cyclin T1, which are viral and cellular proteins. They control gene expression and viral replication. The TAR decoy RNA does not give viral RNA the chance to affect the proteins ${ }^{13}$.

There are two important properties of aptamers that make them ideal candidates for viral diagnosis - their specificity and extremely high affinity for targets. To ensure affinity, SELEX cycles are iterated multiple times with increasing stringency with each cycle. High specificity is a desirable trait for an aptamer. Occasionally, high specificity also causes poor or no cross-reactivity in binding to homologs. A process known as toggle SELEX offers a solution to this problem. In this technique, an RNA pool is allowed to incubate with animal orthologs in addition to the human proteins. This process continues until an aptamer binding to both is selected. White et al. ${ }^{14}$ have used toggle SELEX to ensure cross-reactivity between human and porcine thrombin.

\section{Aptasensors}

Aptamers bind to their targets with a certain specificity, which specificity is determined by the secondary and tertiary structures adapted by these aptamers upon intercalating to the target entity. The intercalating of aptamers to their targets is due to various intermolecular interactions such as pi-pi stacking, hydrogen bonding, van der Waals forces, the involvement of aromatic rings and electrostatic interactions. Biosensors (a combination of bioreceptor and transducer; bioreceptor binds to the target and transducer gives out the biological signals generated by the interaction between the receptor and the target) that employ aptamers are termed as aptasensors (Figure 1$)^{15}$.
Depending on the techniques used and the material to be tested like blood, urine, swabs, etc. aptasensors are classified into two categories: optical aptasensors and electrical aptasensors.

Furthermore, depending on which optical principle is used by which aptasensor, optical aptasensors are classified into various categories ${ }^{15}$.

(1) Chemiluminescence aptasensors: These produce light emission when the aptamer binds to the target due to a chemical reaction called chemiluminescence.

(2) Surface plasmon resonance-based aptasensor: When light of a specific wavelength falls on a metal surface, the free electrons begin to vibrate resulting in surface plasmon resonance. In this technique, the target is deposited on a metal surface. When the aptamer intercalates with the target, the thickness of the metal sheet changes and change in the refractive index is observed. Quantification of the target is done by assessing the intensity of the light polarized ${ }^{16,17}$.

(3) Colorimetric-based aptasensors: When a colorimetric-based aptasensor binds to the target, a change in colour is observed. Colorimetric aptasensors can be used as nanomaterial-assisted or enzyme-linked.

(4) Surface-enhanced Raman scattering (SERS) aptasensors: Nanostructured gold is an example of a SERS active surface. The SERS technique is used for molecular detection, depending on the Raman scattering of molecules adsorbed on the SERS active surface ${ }^{18}$. A SERS-based aptasensor has been developed against the nucleoprotein of influenza virus ${ }^{19}$.

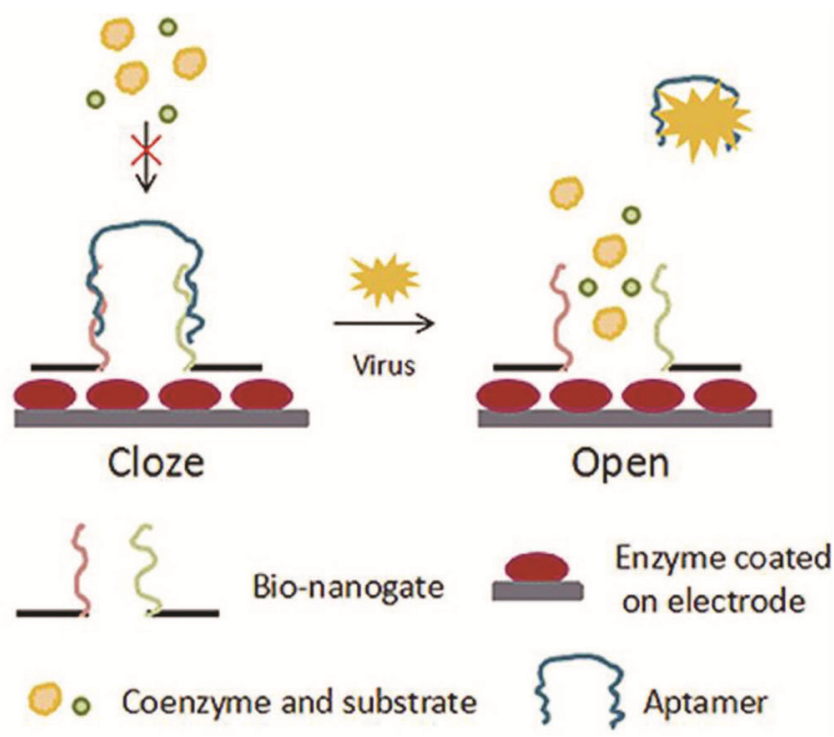

Figure 1. Mechanism of nanogate. When there is no target virus present, the door is closed by an aptamer bound on it. This prevents the enzyme present on the electrode from reacting with any coenzyme and substrate. In the presence of the virus, the aptamer gets attached to it, thus opening the gate. The opening of the gate allows reaction of the enzyme with the coenzyme and substrate, resulting in a change in electrical signal on the electrode. Adapted from Zou et al. ${ }^{15}$. 
(5) Fluorescence aptasensors: These are further of two types, one that changes fluorescent intensity and another that reacts with fluorescence polarization.

(6) Interferometry aptasensors: Using the interferometry technique, we can measure the intensity of a light beam that is produced when different light beams interfere. Hepatitis $\mathrm{C}$ virus (HCV) can be detected using an RNA aptamer technique.

Aptamers bound to the targets either produce an electrical signal or change it, forming the principle behind electrical aptasensors. Based on the working mechanism, they are further classified into electrochemical aptasensors and piezoelectric transducers ${ }^{15,20}$. The former include aptasensors with enzymes, aptasensors without enzymes and FET aptasensors. Aptasensors that do not use enzymes directly result in a change of impedance when the target binds to the aptamers ${ }^{21}$.

The electrical aptasensor with enzymes utilizes nanogates (devices used for controlling reactions). The binding of the virus to the aptamer opens the gate. The opening of the gate causes few other reactions to occur, which in turn produce observable changes ${ }^{22}$. When a particular class of aptamers bind to the target, it results in a change in charge distribution. FET $^{23}$ (a semiconductor device using an electric field to regulate electric behaviour) aptasensors help observe this change (Figure 1).

The aptasensor used to detect human immunodeficiency virus 1 (HIV-1) measures change in the current/nature of the current flowing through the silicon-nitride membrane with a fixed voltage applied at its ends ${ }^{24}$.

\section{Aptamers and antibodies}

Aptamers, the nucleic acid-based binders compare well with their protein-based analogs, i.e. antibodies. Although both aptamers and antibodies show binding affinities in low nanomolar to picomolar range ${ }^{25}$, aptamers are seen as a modern replacement for antibodies, both in therapeutics and diagnostics. Methods which use antibodies as the mean for detection are cumbersome and take several hours to give correct results. For selecting antibodies, we need a biological system. Aptamer selection can be carried out in vitro. This allows aptamer selection in principle against any target of choice ranging from small molecules to macromolecules to cells, currently not possible with antibodies. Some of the benefits of aptamers are: their ability to be stably linked to solid surfaces ${ }^{26}$, their stability under thermally hazardous conditions $^{27}$, ease of synthesis ${ }^{27}$, non-immunogenicity ${ }^{27}$, their ability to undergo chemical modifications and their costeffectiveness making them commercially attractive ${ }^{27,28}$.

\section{Aptamer selection strategies}

The classic strategy for aptamer selection is called SELEX. The process of aptamer selection is similar to the process of antibody selection using phage display in multiple ways $^{26-28}$. The first step in SELEX is obtaining a DNA library of $\sim 10^{15}$ diverse sequences which is then exposed to target entities like the DNA-binding protein of interest. Post incubation, the sequences that intercalate with the protein of interest are separated from the unbound sequences. The next step involves amplification of the sequences bound to the protein of interest by PCR. All the DNA in the vast SELEX library have the same sequence at their ends and random sequences in the middle. Using the primers against the end sequences of the library, the sequence of DNA bound to the protein is amplified and sequenced. Post amplification, a subset of desired oligonucleotides in appropriate numbers is obtained. The entire process is repeated 8-12 times with increasing stringency at each cycle (Figure 2). Numerous iterations are run to ensure high affinity, and to remove nonspecific and weak binders ${ }^{27}$. Multiple strategies have been developed recently that attempt to modify the traditional SELEX procedures to make them more efficient in terms of specificity, affinity, time and cost-effectiveness. Some of them are counter-SELEX and cell-SELEX, capillary electrophoresis SELEX $^{29-42}$.

\section{Aptamers in diagnostics and therapeutics}

Aptamers have established themselves as promising tools that can be used to diagnose and inhibit the progression of a multitude of bacterial and viral infections. Applications of aptamers have been described across the following dimensions: preventing the penetration of antigens into the cells, inhibiting the function of proteins and

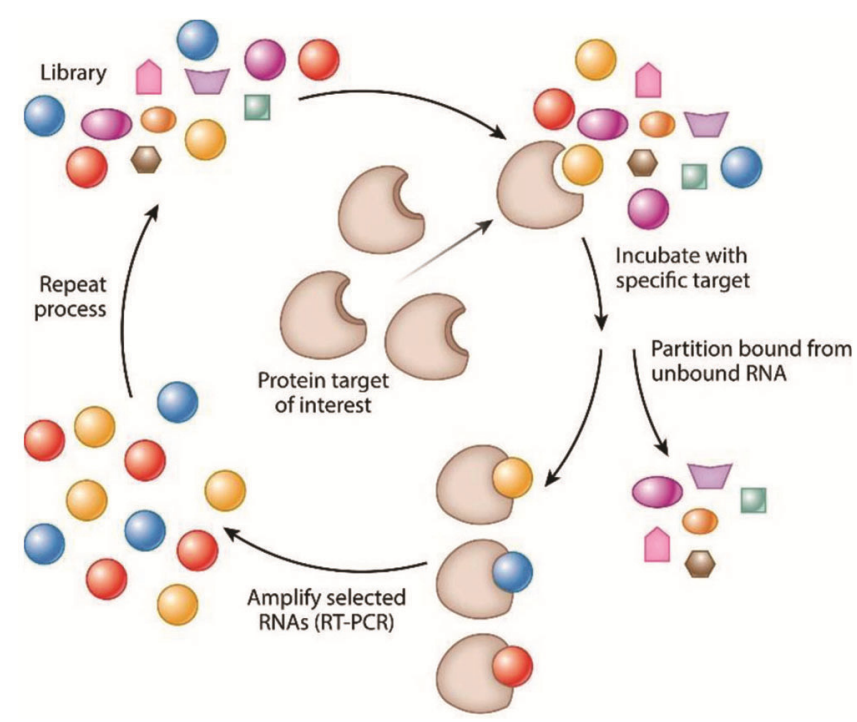

Figure 2. Schematic of the SELEX process to enrich aptamers against any target of choice, such as proteins. The figure represents the steps involved in the process starting from generation of a nucleic acid library of nucleotide sequences to amplifying the selected RNAs. Adapted from Nimjee et al. ${ }^{27}$. 
enzymes that are responsible for viral replication, delivering therapeutic molecules to cells attacked by the virus and selective stimulation of immune functioning. Yet another attractive target is the structural protein of the virus itself ${ }^{43}$.

To the extent of preventing the fusion of the virus within the cells, aptamers have been devised to prevent $\mathrm{HCV}$ infection. The aptamer assigned as ZE2 is bound to the E2 receptors which $\mathrm{HCV}$ glycoprotein uses as an entry point ${ }^{44}$. $\mathrm{Chu}^{45}$ has also used an aptamer-siRNA conjugation approach to establish the therapeutic efficacy of oligonucleotides. The aptamer was linked to the siRNA using streptavidin as a non-covalent bridge to inhibit the gene expression of prostate-specific membrane antigen (PSMA) on LNCaP cells. The aptamer chosen for delivering the target was already known for its binding efficacy to human prostate cancer cells, and its efficacy was proved because the siRNA-mediated gene inhibition was contingent upon its conjugation to the aptamer ${ }^{45}$. Pan et $a l .{ }^{46}$ reviewed how ssDNA/RNA aptamers can target bacterial infections such as Mycobacterium tuberculosis (M tb), Escherichia coli, Salmonella, Listeria monocytogenes and Staphylococcus aureus. They divided aptamers targeting bacteria into two categories: those that target known bacteria-specific surface factors or antigens, and the ones that identify whole cell surfaces without the knowledge of specific molecular targets ${ }^{46}$. For the former category, an ssDNA aptamer was devised against the glycolipid antigen called ManLAM (mannose-capped lipoarabinomannan) of $M t b$ for the diagnosis of tuberculosis (TB). ManLAM is a promising antigen for TB detection as it is released early in the blood circulation system of patients with $M t b$ infection, and also shows immunomodulatory activity. The aptamer named T9 works by detecting this antigen in both the sputum and serum samples of individuals infected with active pulmonary and extrapulmonary $\mathrm{TB}^{47}$. For the latter category of identifying whole cell surfaces, researchers generated aptamers against Listeria monocytogenes over different bacterial growth phases. Out of ten aptamer candidates, four were shown to have a high binding affinity for monocytogenes when selected against five species within the Listeria genus. While three of these DNA aptamers, namely LM6-2, LM12-6 and LM12-13, attached to a single cell moiety, the fourth (LM6-116) targetted another region of the cell surface ${ }^{48}$. While the authors selected the whole surface of mytocyotogenes for aptamer selection, other studies have also used specific proteins of these cells for bacterial detection. For example, Ohk et al. ${ }^{49}$ generated an ssDNA aptamer against the invasion protein of L. monocytogenes called internalin $\mathrm{A}$ as the target molecule.

Aptamers have also exhibited positive results for a spectrum of fatal viral infections such as HCV, HIV-1, Influenza virus, hepatitis B virus (HBC), severe acute respiratory syndrome coronavirus (SARS-CoV), etc.
These authors have also classified aptamers based on whether they target the infected cells expressing structural proteins on the surface (such as ssDNA oligonucleotide GE54 targeting the glycoprotein on the rabies virusinfected cells), or if they target viral enzymes to inhibit their replication (such as an ssDNA aptamer against the SARS-CoV helicase enzyme $)^{46}$. Liang et al. ${ }^{50}$ used cellSELEX technology to generate aptamers against RABV BHK-21 cells and ended up with FO24 and FO21 as final probes. Experimental mice had been infected with the FJ strain of rabies virus; the group that had received preexposure prophylaxis had a higher survival rate than the post-exposure group. Specifically, when the mice had been inoculated with aptamers for $24 \mathrm{~h}$ (through intracranial or intramuscular pathway) before the injection of the FJ strain, the survival rate was $60 \%$ (ref. 50).

Another interesting application of aptamers with respect to selective activation of immune cells is the following: the RNA aptamer CL9 attached itself to a cytosolic receptor retinoic acid inducible gene-1 (RIG-1). This receptor is known to recognize the pattern of foreign invasive molecules. When the CL9 aptamer binds to this receptor, it stimulates the release of IFN-beta, thus selectively activating antiviral immunity ${ }^{51}$.

Despite their promising diagnostic and therapeutic efficacy, aptamers are susceptible to degradation by bodily chemical fluids. However, this can be prevented by adding chemical modifications to the oligonucleotides. Ulrich et al. ${ }^{52}$ suggested that RNA aptamers can acquire more resistance against degradation during the selection and in vivo bodily application by the $2^{\prime}$-fluoro or $2^{\prime}$ amino replacement of $2^{\prime}-\mathrm{OH}$ groups of ribose of pyrimidines. For DNA aptamers, resistance may be achieved by chemically modifying with phosphorothioate ${ }^{52}$. Similarly, the stability of aptamers can be enhanced by capping the 3 '-end with inverted biotin or thymidine after SELEX to prevent their degradation in blood ${ }^{53}$.

\section{Aptamers in COVID-19 research}

Structurally, SARS-CoV-2 comprise a single positive strand of ribonucleic acid which constitutes its genome which is covered by the $\mathrm{N}$ (nucleocapsid) protein (Figure 3 ). The $30 \mathrm{~kb}$ viral genome comprises open reading frame (ORF) $1 \mathrm{a}$ and $1 \mathrm{bb}$ at the $5^{\prime}$-end which encode nonstructural proteins (ppla and pplab). The 3 '-end of the genome encodes essential structural proteins, namely spike glycoprotein (S), envelope protein (E), membrane protein $(\mathrm{M})$, nucleocapsid $(\mathrm{N})$ protein as well as accessory proteins namely, ORF 3, 6, 7a, 7b, 8 and 9b (ref. 54). The virus uses its spike proteins to latch onto the angiotensin converting enzyme (ACE2) receptors which are densely found in lungs and intestine. Zhou et al. ${ }^{55}$, recently reviewed antiviral tools against a multitude of viral targets, such as the spike protein, ACE-2 receptor targets, 
viral replicase enzymes and the viral genome itself ${ }^{55}$. Given the effectiveness of aptamers against various viruses as described above, the report focuses on available aptamer candidates for these viral targets.

The applications of aptamers derived from SELEX have been suggested to be used as an inhibitory target against the ACE-2 receptor. Theoretically, the ssDNA or RNA aptamers can bind to the ACE-2 receptor proteins, blocking the fusion and entry of the SARS-CoV-2 spike protein. These authors have also suggested utilizing aptamers against the entire viral genome by either using siRNA or antisense nucleotide mediated gene silencing ${ }^{56}$. However, there has been negligible output with respect to aptamers against the viral genome or ACE-2 receptors to block the progression of the coronavirus.

\section{Spike protein}

Spike (S) is a structural protein that renders the characteristic spikes on the surface of the SARS-CoV-2 virus. The protein helps the virus enter the host cell by fusing with the plasma membrane. The protein has two major subdomains: S1 and S2. S1 contains a receptor-binding domain (RBD) and is responsible for providing stability during the prefusion state. S2 contains all the components that will be used for the fusion. The presence of a unique furin cleavage site occurring on the boundary of S1/S2 makes this virus unique. The ectodomain of this spike protein is a trimer $160 \AA$ long, having a cross-section and triangular in shape (Figure 4). The trimer exhibits conformational variability, an important characteristic for promoting entry into the host. The trimer has heterogenous N-linked glycans which point out from its surface, serving various purposes. SARS-CoV-2 S possesses 22 $\mathrm{N}$-linked glycosylation sequences per protomer. The uniqueness in the structure is one of the reasons why few of

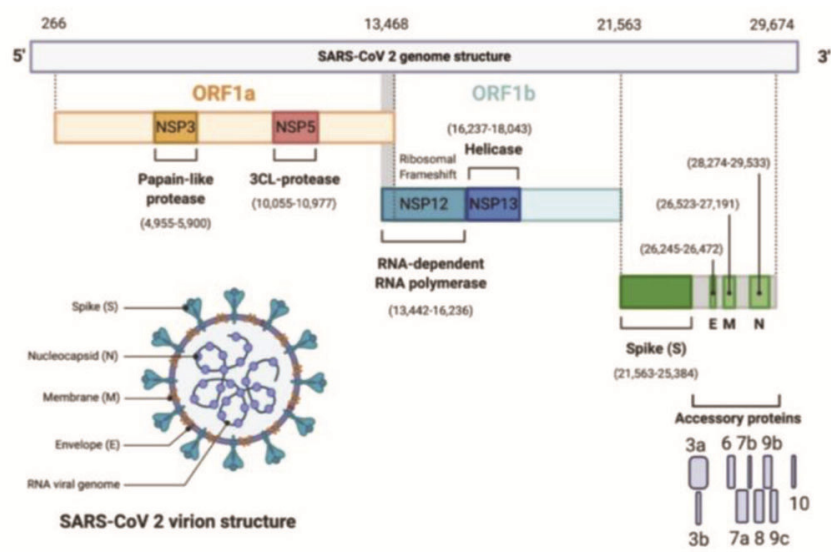

Figure 3. Genomic architecture of SARS-CoV-2. The genome of the virus is $29.9 \mathrm{~kb}$ in length. Two large genes namely $O R F 1 a$ and $O R F 1 b$ encode for the non-structural proteins. The structural proteins (S, E, M and $\mathrm{N}$ ) are encoded by the structural genes. The part shaded grey in the figure codes for accessory proteins. Adapted from Alanagreh et al. ${ }^{82}$. the drugs applicable for other coronaviruses are not effective against this new virus. The host proteases cleave the $\mathrm{S} 2^{\prime}$ site resulting in activation of the protein that causes membrane fusion. The RBD and proteolytic functions promote entry into the cell. After entering inside the body of the host, S proteins are the primary targets of neutralizing antibodies. It has been found that the sera obtained from a mouse infected with SARS-CoV can potentially inhibit the entry of S proteins of SARS-CoV-2 (ref. 57). To the best of our knowledge, no aptamers currently exist against the spike protein of this virus. However, there are aptamers (rich in G-quadruplexes) against the Gp120 glycoprotein of HIV that helps the virus attach onto the host T-helper cells ${ }^{58}$. Similarly, there remains a potential for the discovery of aptamers against the spike glycoprotein of SARS- CoV-2, which is a critical protein for entry into the ACE-2 receptors.

\section{ACE-2 receptors}

The renin-angiotensin system (RAS) has an essential function in controlling renal and cardiovascular functions. Angiotensin-converting enzyme (ACE) is pivotal in regulating RAS. ACE2 is a homolog of ACE, playing an opposing role to $\mathrm{ACE}$ in regulating the renninangiotensin system. A group of four enzymes, each member being commonly referred to as angiotensin (Ang), regulates the blood pressure and overall well-being of the body ${ }^{59,60}$. Ang is converted to Ang I; proteolytic enzyme renin supports the process. Ang I is cleaved by ACE2 to

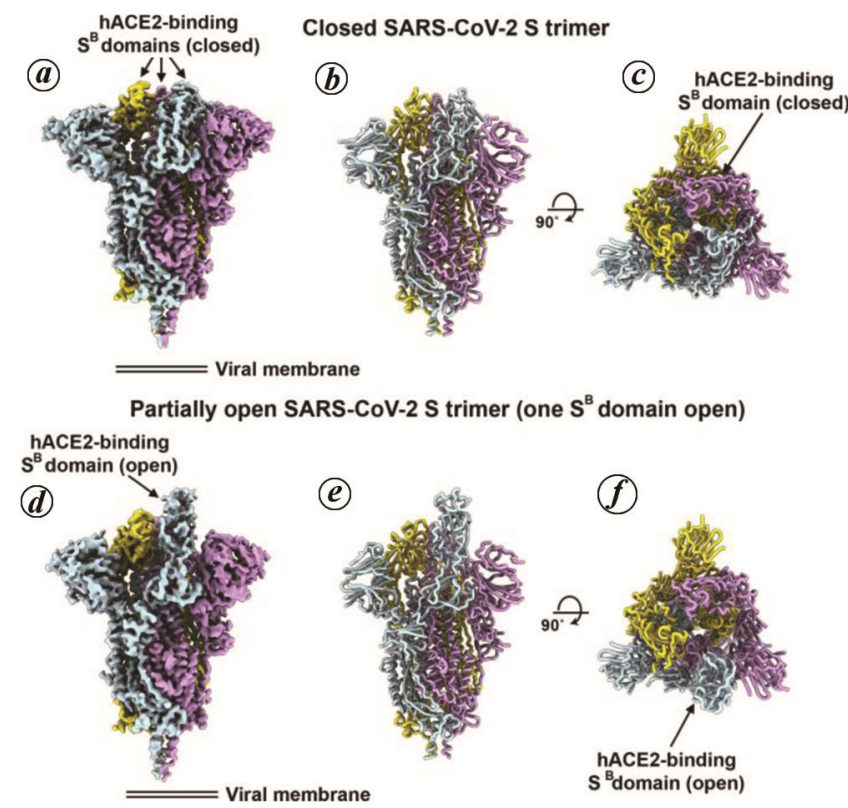

Figure 4. Cryo-EM structures of SARS-CoV-2 S glycoprotein domain for binding to ACE-2 receptors on host cells. $\boldsymbol{a}$, Closed SARSCoV-2 S trimer. $\boldsymbol{b}, \boldsymbol{c}$, Side and top view respectively, of the closed trimer. $\boldsymbol{d}$, Partially open SARS-CoV-2 S trimer. $\boldsymbol{e}, \boldsymbol{f}$, Side and top view respectively of the partially open trimer. Adapted from Walls et al. ${ }^{57}$. 
give Ang II. Ang 1-7 is generated from Ang I when a single residue is cleaved from Ang I using ACE2. A single residue is removed from Ang I with the help of ACE2 to give Ang 1-9.

ACE2 is a type-I transmembrane glycoprotein having 805 amino acids. It possesses an extracellular aminoterminal catalytic domain and a carboxy-terminal domain, and has a molecular weight of $120 \mathrm{kDa}$ (approximately). The enzyme is majorly found in tissues of heart, kidney and testes, and in lower levels in various tissues. In the case of acute lung injury (ALI), it has been found that AngII, ACE and AT1R help in promoting lung injury, whereas ACE2 is involved in a protecting role. ACE2 has been identified as the receptor for SARS coronavirus. SARS-CoV infection causes severe acute respiratory distress syndrome (ARDS), leading to a high mortality rate. ACE2 plays a major role in controlling ARDS after the onset of the disease in the patient. The receptor is also a possible therapeutic and diagnostic $\operatorname{target}^{60}$, but no aptamer exists against the same to date.

\section{E protein}

The coronaviruses are enveloped viruses ${ }^{61}$. In the studies performed on various coronaviruses like infectious bronchitis virus (IBV) and mouse hepatitis virus (MHV), it has been found that coronavirus $\mathrm{E}$ protein is a small integral membrane protein containing 76-109 amino acids $^{62}$. The primary amino acid sequence of the $\mathrm{E}$ protein in SARS-CoV-2 and SARS-CoV shows $94.7 \%$ sequence identity ${ }^{63}$. The $\mathrm{E}$ protein serves numerous purposes. It shows ion-channel activity forming conductive pores in lipid bilayers ${ }^{64}$. The ion channel in SARS-CoV is cationselective $^{65}$. This allows entry of calcium into the Golgi membrane complexes/endoplasmic reticulum, which leads to the activation of the NLRP3 inflammasome and triggers the production of interleukin- $\beta$. The protein is also implicated in viral assembly, viral pathogenesis and virion release $\mathrm{e}^{63}$. The membrane structure of $\mathrm{E}$ protein is still not completely clear, but many claim that it is a transmembrane protein having $\mathrm{N}$-terminal ectodomain and C-terminal endodomain ${ }^{66}$. Various studies provide different reports while declaring the proteins involved in the envelope formation. Findings state that either $M$ and $\mathrm{N}, \mathrm{M}$ and $\mathrm{E}$ or $\mathrm{M}$ alone can cause envelope formation. It suppresses the host-cell response. It has also been found that SARS-CoV E can be anti-apoptotic in the infected individual $^{62}$. Figure 5 is a cartoon representation of the SARS-CoV-2 structural proteins. No aptamers have been developed against the E protein of SARS-CoV-2 to the best of our knowledge, although Chen et al. ${ }^{44}$ have developed aptamers against the envelope glycoprotein (E2) found in human $\mathrm{HCV}$. Such findings should catalyse scientific efforts to devise aptamers against the E protein of SARS-CoV-2 as well.

\section{M protein}

The $\mathrm{M}$ protein has a vital role in the assembly of the coronavirus. It is responsible for the very shape of the virion. It is a structural protein, quite abundant in the virion. It has an $\mathrm{N}$-terminal ectodomain and a $\mathrm{C}$-terminal endodomain $^{66}$. The $\mathrm{M}$ protein appears like tightly packed lines over the membrane and touching the ribonucleoprotein (RNP). The protein is known to exhibit two conformations which help it in binding to the nucleocapsid. The functional form of the protein is a homodimer ${ }^{67}$. Most of the information available on $\mathrm{M}$ protein is related to the $\mathrm{M}$ protein of various coronaviruses like mouse hepatatis virus or infectious bronchitis virus. Since the $M$ proteins in most of the coronaviruses resemble each other, similar conclusions can be drawn for the M protein of SARS-CoV 2. However, no aptamers exist against the $\mathrm{M}$ protein of SARS- CoV-2 till date.

\section{$N$ (Nucleocapsid) protein}

The coronavirus $\mathrm{N}$ protein is an RNA-binding protein serving multiple functions. It is integral for transcription and replication of viral RNA. It plays a vital role in packing the viral RNA genome by forming helical RNPs. The protein is also involved in modulating the mechanism of the infected cell.

Three distinct domains make up this protein: (i) C-terminal dimerization domain for oligomerization.

(ii) N-terminal RNA-binding domain for RNA binding. (iii) Intrinsically disordered central Ser/Arg linker for primary phosphorylation.

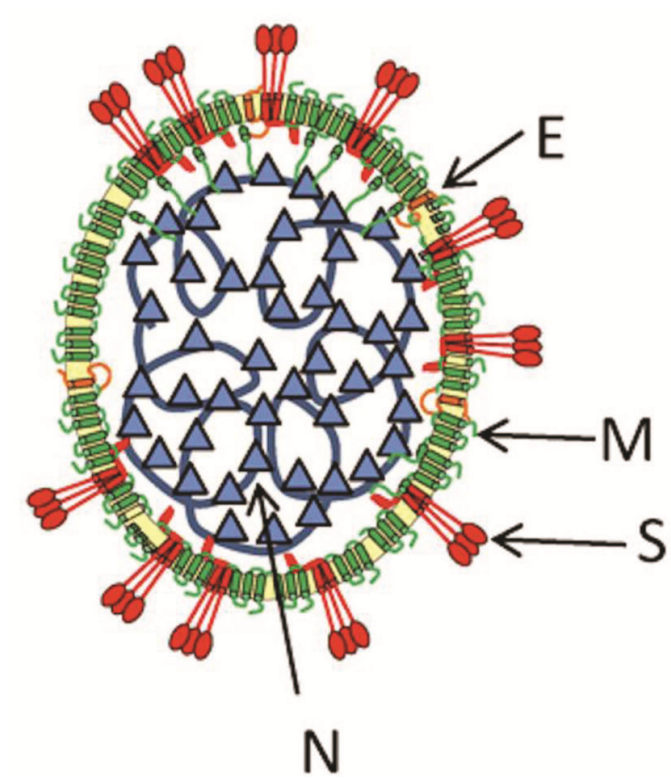

Figure 5. Cartoon representation of coronavirus virion, representing the four structural proteins, viz. envelope protein (E), spike protein (S), membrane protein $(\mathrm{M})$ and nucleocapsid protein $(\mathrm{N})$. Adapted from Ruch and Machamer ${ }^{62}$. 
In SARS-CoV-2, the $3^{\prime}$-end ORF (open reading frame) encodes for the nucleocapsid protein. Understanding the structure of the SARS-CoV-2 N-NTD (the N-terminal region of $\mathrm{N}$ protein of SARS-CoV-2 virus) will help in studying the molecular interactions when it binds to ribonucleotides, blocking viral assembly and multiplication. SARS-CoV-2 N-NTD crystals pack in an orthorhombic fashion. All the four monomers present in a crystal have a right-handed sandwiched fold (loops-beta sheet coreloops). SARS-CoV-2 N-NTD has many aromatic and basic residues in its structure. The overall organization of various coronavirus $\mathrm{N}$ proteins is similar. It is the surface charge distribution that distinguishes one from the other type (Figure 6$)^{54}$.

The $\mathrm{N}$ protein is highly immunogenic and highly expressed when the infection occurs. It is, therefore, an important viral protein that should be studied in more detail $^{54}$. High-affinity aptamer has been selected that binds to the $\mathrm{N}$ protein of the SARS-CoV responsible for the SARS-CoV 2002-2004 outbreak. Using the selected aptamer as the antigen-capturing agent in chemiluminescence immunosorbent assay and nanoarray aptamer chip, low levels of $\mathrm{N}$ protein as low as $2 \mathrm{pg} / \mathrm{ml}$ could be detected $^{25}$. A nanoarray aptamer chip method is based on hybridization technique. In this method, a nanoarray is constructed using microfabrication tools and aptamers are immobilized onto these arrays for capturing the protein of interest (in this case $\mathrm{N}$ protein of SARs CoV), which can be detected by utilizing various detection techniques ${ }^{68}$. Using the classical SELEX protocol, an ssDNA aptamer binding with high affinity and specificity to the SARS$\mathrm{CoV} N$ protein has been recently reported ${ }^{69}$.

\section{Helicase enzyme}

ORF1a and ORF1b of the viral genome are translated into two large replicative polyproteins (ppla and pplab) using ribosomal frameshifting. These polyproteins are further processed into 16 non-structural proteins through the process of auto-proteolysis. Two non-structural proteins,

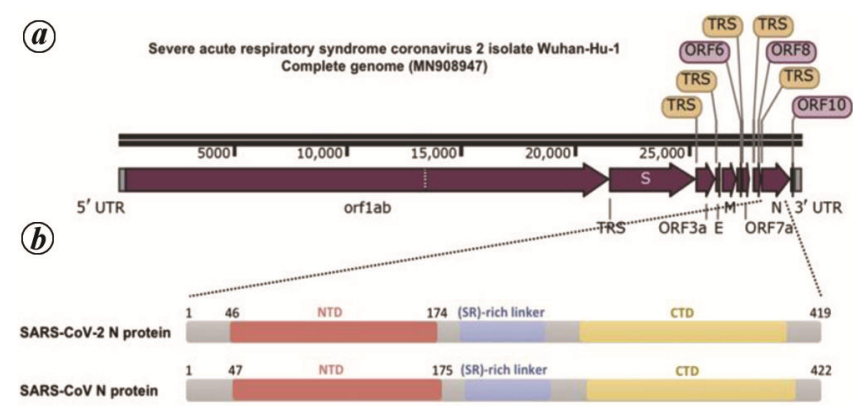

Figure 6. a, Whole genomic features from SARS-CoV-2 isolate UTR, Untranslated region; ORF, Open reading frame; TRS, Transcriptional regulatory sequence; S, M, E, N, Encoding region of the respective proteins. $\boldsymbol{b}$, Domain architecture of $\mathrm{N}$ protein of SARS-CoV-2 N protein and SARS-CoV. Adapted from Kang et al. ${ }^{54}$. viz. RNA-dependent RNA polymerase (nsp12) and NTPase/helicase (nsp13) are considered to constitute the membrane-bound transcription-replication complex ${ }^{70}$. The SARS-CoV helicase comprises three principal domains: N-terminal membrane binding domain, a hinge and an NTPase/helicase domain. The helicase enzymes are motor proteins that are responsible for unwinding the double-stranded nucleic acids utilizing the energy from the hydrolysis of nucleotides ${ }^{71}$. These authors have also reviewed a range of other functions performed by the helicase enzyme: displacement of proteins from nucleic acids, remodelling of chromatin structure, displacement of Holliday junctions and various facets of RNA metabolism like transcription, splicing, export of mRNA and gene expression in the mitochondria. Although the exact role of helicase in viral replication is not fully understood, most antiviral strategies against this enzyme have been targeted to stop its unwinding activity. One probable mechanism through which the helicase enzyme could facilitate genome replication is through the unwinding of the knotted/folded RNA (due to its great length), allowing the transcription/replication of RNA-dependent RNA polymerase. Additionally, the role of helicase enzymes in unfolding the G-quadruplex structures is known. The Gquadruplex structures are non-canonical tetra-helical structures that come into existence by the folding of guanine-rich sequences. These folded structures pose a challenge to the replication, transcriptional and translational processes. Thus, nucleic acids need kinetic forces that act upon these tightly folded G-quadruplexes, so that metabolic processes like replication can follow ${ }^{72}$. Most polymerases do not have adequate energy to unwind this structure. Therefore, eukaryotic cells have evolved to use helicase enzymes for unwinding these quadruplexes to initiate replication (the exact mechanism by which helicase unfolds these quadruplex structures remains inadequately known). However, helicase remains an attractive antiviral target because these G-4 structures are found in many viral genomes, including SARS-CoV-2. Identifying specific vial proteins is of paramount importance to find suitable inhibitors against them. In this regards, using antibody labelling assay in cells infected with coronavirus, Prentice et $a l^{73}$ identified and characterized the replicase protein in SARS-CoV virus. Therefore, efforts have been made towards devising measures to inhibit helicase activity. For instance, Cho and colleagues generated a small compound EMMDPD (7-ethyl-8-mercapto-3-methyl-3,7dihydro-1H-purine-2, 6-dione). This compound was able to impede adenosine triphosphate hydrolysis as well as the unwinding activity of the helicase. The compound had an advantage in the sense that it did not lead to any cellular cytotoxicity ${ }^{74}$.

In terms of aptamers, however, there are few studies on how these decoys could be used to inhibit helicase activity. For example, Shum and Tanner ${ }^{53}$ reported the differential features of G-quadruplex and non-G quadruplex 
DNA aptamers in inhibiting the unwinding activity of SARS-CoV helicase enzyme. They incubated the DNA oligonucleotides with Ni-NTA beads using SELEX technology. While all of five selected candidates actively stimulated ATPase activity and showed high affinity for helicase, only the non-G quadruplex class of aptamers could halt the unwinding function of helicase, suggesting that only these structures were capable of locking the nucleic acid-binding sites into high ATPase turnover conformation, whereas G-quadruplex aptamers could not ${ }^{53}$. Jang et al. $^{75}$ developed RNA aptamers against SCV (SARS coronavirus) NTPase/helicase to inhibit the unwinding activity of helicase enzyme, which was found to show efficient activity up to $\sim 85 \%$ with an $\mathrm{IC}_{50}$ value of $1.2 \mathrm{nM}$. However, it had a lower effect on the ATPase activity of the protein along with the presence of poly $(\mathrm{rU})^{75}$. These findings form a basis of further exploration of aptamers against the helicase enzyme of SARS-Cov-2 as well.

\section{RNA-dependent RNA polymerase}

The RNA-dependent RNA polymerase (RdRps) (nsp12) is an integral enzyme in the viral genome transcriptionreplication machinery. In addition to this enzyme two cofactors, namely nsp 7 and nsp 8 have been found to be essential components when nsp12 binds to initiate genome replication $^{76}$. Analogous to other RdRps, nsp12 of SARS-Cov-2 adopts a structure like that of a right hand with the finger, palm and thumb subdomains. RdRp has already been established as the primary target for the antiviral drug remdesivir ${ }^{77}$. This is a nucleotide analog that causes the Rdrp to recognize and incorporate the active form of this inhibitor drug, and the consequent binding prevents the polymerase from replicating the viral genome further. Due to its efficacy in symptom reduction $^{78}$, and no consequent adverse reactions ${ }^{79}$, favipiravir is also considered as an attractive antiviral drug.

No aptamers are reported against the Rdrp of SARSCoV-2. Although there have been aptamers devised against the RdRp (NS5B) of HCV. Like in SARS-CoV-2, $\mathrm{RdRp}$ is essential for the generation of positive-sense genomic RNA and replicating a negative-sense template from the same. For example, Bellecave et al. ${ }^{80}$ developed DNA aptamer against the HCV NS5B protein, which competed with the positive and negative-strands of HCV viral RNA so as to bind RdRp to itself and block the initiation and elongation of transcription of viral RNA. Similarly, research should focus on designing aptamers against the nsp13 of SARS-CoV-2, because most RdRps share structural similarity in terms of consisting of a finger, thumb and palm domain. Additionally, while smallmolecule drugs such as HCV protease inhibitors or HIV integrase inhibitors attack the active sites of the enzyme to impede their catalytic role, aptamers are additionally able to bind more tightly to the protruding parts of the protein, thus preventing protein-protein interactions ${ }^{81}$. SARS-CoV-2 RdRp nsp13 also interacts with nsp 7 and nsp 8 to initiate its replication activity. Therefore, aptamers should be tested against nsp13 because of their proven efficacy in inhibiting protein-protein interactions.

\section{Conclusion and perspectives}

Here we present a brief overview of the DNA aptamers and the methods involved with special focus to the development of aptamers against viral components. The field of aptamers presents much more advantages over antibodies in terms of specificity, affinity, minimum immunogenicity and cost-effectiveness in terms of production, modifications and applications. Though aptamers have been evolved and developed for various viruses such as HIV, HCV and SARS-CoV, we still need a large palette of aptamers for the genomic RNA and multiple proteins of SARS-CoV-2. Also, at present, the aptamers are mostly restricted as sensing modules, but they also have the capacity to be used as inhibitory drugs. This aspect of aptamers still remains to be largely explored. We can foresee immense developments in the field of aptamers, where the next goals would be to develop multimodal aptamers with capability to recognize and inhibit the targets. This would definitely be of immense use in therapeutics, especially in pandemic infections like COVID-19.

Conflict of interest: The authors declare no conflict of interest.

1. Scagnolari, C., Turriziani, O., Monteleone, K., Pierangeli, A. and Antonelli, G., Consolidation of molecular testing in clinical virology. Expert. Rev. Anti.-Infect Ther., 2017, 15(4), 387-400.

2. Storch, G. A., Diagnostic virology. Clin. Infect. Dis., 2000, 31(3), 739-751.

3. Jacofsky, D., Jacofsky, E. M. and Jacofsky, M., Understanding antibody testing for COVID-19. J. Arthroplasty, 2020, 35(7S), S74-S81.

4. Peaper, D. R. and Landry, M. L., Laboratory diagnosis of viral infection. Handbook of Clinical Neurology, Sciencedirect, Elsevier, 2014, vol. 123, pp. 123-147.

5. Murin, C. D., Wilson, I. A. and Ward, A. B., Antibody responses to viral infections: a structural perspective across three different enveloped viruses. Nature Microbiol., 2014, 4(5), 734-747.

6. Flyak, A. I. et al., Cross-reactive and potent neutralizing antibody responses in human survivors of natural Ebola virus infection. Cell, 2016, 164(3), 392-405.

7. Bustin, S. A. and Mueller, R., Real-time reverse transcription PCR (qRT-PCR) and its potential use in clinical diagnosis. Clin. Sci., 2005, 109(4), 365-379.

8. Mullis, K. B., The unusual origin of the polymerase chain reaction. Sci. Am., 1990, 262(4), 56-65.

9. Netzer, K. O., Hybridization methods (southern and northern blotting). In Techniques in Molecular Medicine (eds Hildebrandt, F. and Igarashi, P.), Springer Lab Manual, Springer, Berlin, Germany, 1990, pp. 126-147. 
10. $\mathrm{Ku}, \mathrm{T} . \mathrm{H}$. et al., Nucleic acid aptamers: an emerging tool for biotechnology and biomedical sensing. Sensors, 2015, 15(7), $16281-16313$

11. Zhu, Q., Liu, G. and Kai, M., DNA aptamers in the diagnosis and treatment of human diseases. Molecules, 2015, 20(12), 20979 20997.

12. Tuerk, C. and Gold, L., Systematic evolution of ligands by exponential enrichment: RNA ligands to bacteriophage T4 DNA polymerase. Science, 1990, 249(4968), 505-510.

13. Sullenger, B., Gallardo, H., Ungers, G. and Gilboa, E., Analysis of trans-acting response decoy RNA-mediated inhibition of human immunodeficiency virus type 1 transactivation. J. Virol., 1991, 65, 6811-6816.

14. White, R., Rusconi, C., Scardino, E., Wolberg, A., Lawson, J., Hoffman, M. and Sullenger, B., Generation of species crossreactive aptamers using 'toggle' SELEX. Mol. Therapy, 2001, 4, $567-573$.

15. Zou, X., Wu, J., Gu, J., Shen, L. and Mao, L., Application of aptamers in virus detection and antiviral therapy. Front. Microbiol., 2019, 10, 1462.

16. Nguyen, V., Seo, H. B., Kim, B. C., Kim, S. K., Song, C. S. and $\mathrm{Gu}, \mathrm{M}$. B., Highly sensitive sandwich-type SPR based detection of whole $\mathrm{H} 5 \mathrm{Nx}$ viruses using a pair of aptamers. Biosens. Bioelectron., 2016, 86, 293-300.

17. Homola, J. and Piliarik, M., Surface plasmon resonance (SPR) sensors. In Surface Plasmon Resonance Based Sensors (ed. Homola, J.), Springer Series on Chemical Sensors and Biosensors, Springer, Berlin, Germany, 2006, vol. 4, pp. 45-67.

18. Sharma, B., Frontiera, R. R., Henry, A.-I., Ringe, E. and Van Duyne, R. P., SERS: materials, applications, and the future. Mater. Today, 2012, 15, 16.

19. Negri, P., Chen, G., Kage, A., Nitsche, A., Naumann, D., Xu, B. and Dluhy, R. A., Direct optical detection of viral nucleoprotein binding to an anti-influenza aptamer. Anal. Chem., 2012, 84, 5501-5508.

20. Cataldo, R., Leuzzi, M. and Alfinito, E., Modelling and development of electrical aptasensors: a short review. Chemosensors, 2018, 6, 20

21. Lum, J., Wang, R., Hargis, B., Tung, S., Bottje, W., Lu, H. and Li, Y., An impedance aptasensor with microfluidic chips for specific detection of H5N1 avian influenza virus. Sensors, 2015, 15, 18565-18578.

22. Fu, Y., Callaway, Z., Lum, J., Wang, R., Lin, J. and Li, Y., Exploiting enzyme catalysis in ultra-low ion strength media for impedance biosensing of avian influenza virus using a bare interdigitated electrode. Anal. Chem., 2014, 86, 1965-1971.

23. Rahim Ruslinda, A., Tanabe, K., Ibori, S., Wang, X. and Kawarada, H., Effects of diamond-FET-based RNA aptamer sensing for detection of real samples of HIV-1 Tat protein. Biosens. Bioelectron., 2013, 40, 277-282.

24. Niedzwiecki, D., Iyer, R., Borer, P. and Movileanu, L., Correction to sampling a biomarker of the human immunodeficiency virus across a synthetic nanopore. ACS Nano., 2013, 7, 3341-3350.

25. Ahn, D. et al., RNA aptamer-based sensitive detection of SARS coronavirus nucleocapsid protein. Analyst, 2009, 134, 1896.

26. Brody, E. and Gold, L., Aptamers as therapeutic and diagnostic agents. Rev. Mol. Biotechnol., 2000, 74, 5-13.

27. Nimjee, S., White, R., Becker, R. and Sullenger, B., Aptamers as therapeutics. Annu. Rev. Pharmacol. Toxicol., 2017, 57, 61-79.

28. Song, K., Lee, S. and Ban, C., Aptamers and their biological applications. Sensors, 2012, 12, 612-631.

29. Kong, H. and Byun, J., Nucleic acid aptamers: new methods for selection, stabilization, and application in biomedical science. Biomol. Ther., 2013, 21, 423-434.

30. Jenison, R., Gill, S., Pardi, A. and Polisky, B., High-resolution molecular discrimination by RNA. Science, 1994, 263, 14251429 .
31. Mosing, R. K., Mendonsa, S. D. and Bowser, M. T., Capillary electrophoresis-SELEX selection of aptamers with affinity for HIV-1 reverse transcriptase. Anal. Chem., 2005, 77, 6107-6112.

32. Wang, B., A new design for the fixed primer regions in an oligonucleotide library for SELEX aptamer screening. Front. Chem., 2020,8 .

33. Zhong, Y., Zhao, J., Li, J., Liao, X. and Chen, F., Advances of aptamers screened by cell-SELEX in selection procedure, cancer diagnostics and therapeutics. Anal. Biochem., 2020, 598, 113620.

34. Mayer, G., Ahmed, M. S. L., Dolf, A., Endl, E., Knolle, P. A. and Famulok, M., Fluorescence-activated cell sorting for aptamer SELEX with cell mixtures. Nature Protoc., 2010, 5, 1993-2004.

35. Hung, L., Wu, H., Hsieh, K. and Lee, G., Microfluidic platforms for discovery and detection of molecular biomarkers. Microfluid. Nanofluid., 2014, 16, 941-963.

36. Liu, Q. et al., SELEX tool: a novel and convenient gel-based diffusion method for monitoring of aptamer-target binding. J. Biol. Eng., 2020, 14, 1 .

37. Yu, F., Li, H., Sun, W., Xu, D. and He, F., Rapid selection of aptamers based on protein microarray. RSC Adv., 2019, 9, 9762 9768.

38. Zumrut, H. E., Batool, S., Argyropoulos, K. V., Williams, N., Azad, R. and Mallikaratchy, P. R., Integrating ligand-receptor interactions and in vitro evolution for streamlined discovery of artificial nucleic acid ligands. Mol. Therapy-Nucleic Acids, 2019, 17, 150-163.

39. Li, Y., Liu, B., Huang, Z. and Liu, J., Engineering base-excised aptamers for highly specific recognition of adenosine. Chem. Sci., 2020, 11, 2735-2743

40. Kushwaha, A., Takamura, Y., Nishigaki, K. and Biyani, M., Competitive non-SELEX for the selective and rapid enrichment of DNA aptamers and its use in electrochemical aptasensor. Sci. Rep., 2019, 9(1), 1-11.

41. Nutiu, R. and Li, Y., Aptamers with fluorescence-signaling properties. Methods, 2005, 37, 16-25.

42. Yoshitomi, T., Wayama, F., Kimura, K., Wakui, K., Furusho, H. and Yoshimoto, K., Screening of DNA signaling aptamer from multiple candidates obtained from SELEX with next-generation sequencing. Anal. Sci., 2019, 35, 113-116.

43. Wandtke, T., Woźniak, J. and Kopiński, P., Aptamers in diagnostics and treatment of viral infections. Viruses, 2015, 7, 751-780.

44. Chen, F., Hu, Y., Li, D., Chen, H. and Zhang, X., CS-SELEX generates high-affinity ssDNA aptamers as molecular probes for hepatitis C virus envelope glycoprotein E2. PLoS ONE, 2009, 4, e8142.

45. Chu, T., Aptamer mediated siRNA delivery. Nucleic Acids Res., 2006, 34, e73-e73.

46. Pan, Q., Luo, F., Liu, M. and Zhang, X., Oligonucleotide aptamers: promising and powerful diagnostic and therapeutic tools for infectious diseases. J. Infect., 2018, 77, 83-98.

47. Tang, X. et al., Generation and application of ssDNA aptamers against glycolipid antigen ManLAM of Mycobacterium tuberculosis for TB diagnosis. J. Infect., 2016, 72, 573-586.

48. Suh, S., Dwivedi, H., Choi, S. and Jaykus, L., Selection and characterization of DNA aptamers specific for Listeria species. Anal. Biochem., 2014, 459, 39-45.

49. Ohk, S., Koo, O., Sen, T., Yamamoto, C. and Bhunia, A., Antibody-aptamer functionalized fibre-optic biosensor for specific detection of Listeria monocytogenes from food. J. Appl. Microbiol., 2010, 109, 808-817.

50. Liang, H., Liang, H. R., Hu, G. Q., Li, L., Gao, Y. W., Yang, S. T. and Xia, X. Z., Aptamers targeting rabies virus-infected cells inhibit street rabies virus in vivo. Int. Immunopharmacol., 2014, 21, $432-438$.

51. Hwang, S. et al., 5'-triphosphate-RNA-independent activation of RIG-I via RNA aptamer with enhanced antiviral activity. Nucl. Acids Res., 2011, 40, 2724-2733. 


\section{REVIEW ARTICLE}

52. Ulrich, H., Trujillo, C. A., Nery, A. A., Alves, J. M., Majumder, P., Resende, R. R. and Martins, A. H., DNA and RNA aptamers: from tools for basic research towards therapeutic applications. Comb. Chem. High Throughput Screen., 2006, 9, 619-632.

53. Shum, K. and Tanner, J., Differential inhibitory activities and stabilisation of DNA aptamers against the SARS coronavirus helicase. ChemBioChem, 2008, 9, 3037-3045.

54. Kang, S. et al., Crystal structure of SARS-CoV-2 nucleocapsid protein RNA binding domain reveals potential unique drug targeting sites. Acta Pharm. Sin. B, 2020, 10(7), 1228-1238.

55. Zhou, H., Fang, Y., Xu, T., Ni, W. J., Shen, Z. and Meng, X. M., Potential therapeutic targets and promising drugs for combating SARS-CoV-2. Br. J. Pharmacol., 2020, 177, 3147-3161.

56. Rossi, J. and Rossi, D., Oligonucleotides and the COVID-19 pandemic: a perspective. Nucl. Acid Ther., 2020, 30, 129-132.

57. Walls, A., Park, Y. J., Tortorici, M. A., Wall, A., McGuire, A. T. and Veesler, D., Structure, function, and antigenicity of the SARSCoV-2 spike glycoprotein. Cell, 2020, 181, 281-292.

58. Musumeci, D., Riccardi, C. and Montesarchio, D., G-quadruplex forming oligonucleotides as anti-HIV agents. Molecules, 2015, 20, 17511-17532.

59. Eriksson, U., Danilczyk, U. and Penninger, J., Just the beginning: novel functions for angiotensin-converting enzymes. Curr. Biol., 2002, 12, R745-R752.

60. Imai, Y., Kuba, K., Ohto-Nakanishi, T. and Penninger, J., Angiotensin-converting enzyme 2 (ACE2) in disease pathogenesis. Circ. J., 2010, 74, 405-410.

61. Masters, P., The molecular biology of coronaviruses. Adv. Virus Res., 2006, 66, 193-292.

62. Ruch, T. and Machamer, C., The coronavirus E protein: assembly and beyond. Viruses, 2012, 4, 363-382.

63. Yoshimoto, F. K., The proteins of severe acute respiratory syndrome coronavirus-2 (SARS CoV-2 or n-COV19), the cause of COVID-19. Protein J., 2020, 39, 198-216.

64. Verdiá-Báguena, C. et al., Coronavirus E protein forms ion channels with functionally and structurally-involved membrane lipids. Virology, 2012, 432, 485-494.

65. Wilson, L., Mckinlay, C., Gage, P. and Ewart, G., SARS coronavirus $\mathrm{E}$ protein forms cation-selective ion channels. Virology, 2004, 330, 322-331.

66. Fehr, A. and Perlman, S., Coronaviruses: an overview of their replication and pathogenesis. Coronaviruses, 2015, 1282, 1-23.

67. Neuman, B. et al., A structural analysis of M protein in coronavirus assembly and morphology. J. Struct. Biol., 2011, 174, 1122.

68. Mosher, C. Nano arrays, the next generation molecular array format for high throughput proteomics, diagnostics and drug discover. J. Assoc. Lab. Autom., 2000, 5, 75-78.

69. Cho, S., Woo, H., Kim, K., Oh, J. and Jeong, Y., Novel system for detecting SARS coronavirus nucleocapsid protein using an ssDNA aptamer. J. Biosci. Bioeng., 2011, 112, 535-540.

70. van Hemert, M., Van Den Worm, S. H., Knoops, K., Mommaas, A. M., Gorbalenya, E. and Snijder, E. J., SARS-coronavirus replication/transcription complexes are membrane-protected and need a host factor for activity in vitro. PLoS Pathog, 2008, 4(5), e1000054
71. Adedeji, A. et al., Mechanism of nucleic acid unwinding by SARS-CoV helicase. PLoS ONE, 2012, 7, e36521.

72. Mendoza, O., Bourdoncle, A., Boulé, J., Brosh, R. and Mergny, J., G-quadruplexes and helicases. Nucleic Acids Res., 2016, 44, 1989-2006.

73. Prentice, E., McAuliffe, J., Lu, X., Subbarao, K. and Denison, M., Identification and characterization of severe acute respiratory syndrome coronavirus replicase proteins. J. Virol., 2004, 78, 9977-9986.

74. Cho, J., Lee, J., Ahn, H. and Jeong, Y., Identification of a novel small molecule inhibitor against SARS coronavirus helicase. J. Microbiol. Biotechnol., 2015, 25, 2007-2010.

75. Jang, K., Lee, N., Yeo, W., Jeong, Y. and Kim, D., Isolation of inhibitory RNA aptamers against severe acute respiratory syndrome (SARS) coronavirus NTPase/Helicase. Biochem. Biophys. Res. Commun., 2008, 366, 738-744.

76. Gao, Y. et al., Structure of the RNA-dependent RNA polymerase from COVID-19 virus. Science, 2020, 368, 779-782.

77. Yin, W. et al., Structural basis for the inhibition of the RNAdependent RNA polymerase from SARS-CoV-2 by remdesivir. Science, 2020, 368, 1499-1504.

78. Chen, C. et al., Favipiravir versus arbidol for COVID-19: a randomized clinical trial, 2020; doi:org/10.1101/2020.03.17. 20037-432.

79. Dong, L., Hu, S. and Gao, J., Discovering drugs to treat coronavirus disease 2019 (COVID-19). Drug Discov. Ther., 2020, 14, 58-60.

80. Bellecave, P., Andreola, M. L., Ventura, M., Tarrago-Litvak, L., Litvak, S. and Astier-Gin, T., Selection of DNA aptamers that bind the RNA-dependent RNA polymerase of hepatitis $\mathrm{C}$ virus and inhibit viral RNA synthesis in vitro. Oligonucleotides, 2003, 13, 455-463.

81. Shum, K., Zhou, J. and Rossi, J., Aptamer-based therapeutics: new approaches to combat human viral diseases. Pharmaceuticals, 2013, 6, 1507-1542.

82. Alanagreh, L. et al., The human coronavirus disease COVID-19: its origin, characteristics, and insights into potential drugs and its mechanisms. Pathogens, 2020, 9, 331; doi:10.3390/pathogens9050331 .

ACKNOWLEDGEMENTS. We thank e-Summer Research Internship Program at India Institute of Technology Gandhinagar (IITGN) for the research opportunity to S.J. and S.P.S. under the guidance of D.B. D.B. thanks SERB-Ramanujan Fellowship and IITGN start-up grant for financial support. We also thank our faculty colleagues, students and staff at IITGN for stimulating discussions and feedback during the eSRIP project and during the preparation of this manuscript and members of D.B. and S.M. labs at for critically reading the manuscript and providing useful feedback

Received 30 June 2020; revised accepted 27 August 2020

doi: $10.18520 / \mathrm{cs} / \mathrm{v} 119 / \mathrm{i} 9 / 1489-1498$ 